\title{
Wireless Control of Magnetic Helical Microrobots using a Rotating-Permanent-Magnet Manipulator
}

\author{
Thomas W. R. Fountain, Prem V. Kailat, and Jake J. Abbott
}

\begin{abstract}
Magnetic helical medical microrobots are promising for use in open fluid, lumen, and soft-tissue environments and will enable minimally invasive access to hard-to-reach locations in the body. Prior work focused on control via uniform magnetic fields from orthogonal arrangements of electromagnetic coils, which are difficult to scale up to the size required for clinical applications. We propose the use of nonuniform magnetic fields emanating from a single rotating-permanentmagnet (RPM) manipulator for the control of magnetic helical microrobots. We theoretically and experimentally demonstrate that the RPM manipulator is a viable option for wireless control of magnetic helical microrobots that warrants further investigation.
\end{abstract}

\section{INTRODUCTION}

Medical microrobots will enable minimally invasive access to many hard-to-reach locations in the body. They will perform tasks that are currently difficult or impossible, and even some tasks not yet conceived. They have the potential to be used for a wide range of applications including targeted therapy (e.g. drugs, hyperthermia), material removal (e.g. ablation), remote sensing, and as controllable structures (e.g. stents), and they can be used in a number of systems in the human body (e.g. circulatory system, urinary system, central nervous system). A survey of research in wireless medical microrobots can be found in [1]. In this paper, we use "microrobot" for lack of a better term. For many envisioned medical applications, devices may be as large as a few millimeters [1].

Helical propulsion using magnetic fields is particularly promising for medical microrobots. Helical microrobots use a swimming technique inspired by the propulsion of bacterial flagella, which is well suited to a low-Reynolds-number regime [2]. A rotating magnetic field wirelessly generates torque on the microrobot, resulting in rotation of the helix about its axis, coupled with forward/backward propulsion. The helical propellers can take the form of corkscrew-like structures or wood-screw-like structures, with propulsion and control being very similar in both cases. A research group at Tohoku University was the first to investigate magnetic helical microrobots propelled by rotating magnetic fields. They have experimented with corkscrew-like devices [3], as well as devices shaped like wood screws [4]. In each case, a permanent magnet is rigidly attached to the propeller, with its magnetization perpendicular to the axis of the helix. The Tohoku group has explored radio-frequency heating of the microrobots for hyperthermia therapy [5], [6], as well

The authors are with the Department of Mechanical Engineering, University of Utah, Salt Lake City, UT 84112, USA. jake.abbott@utah.edu

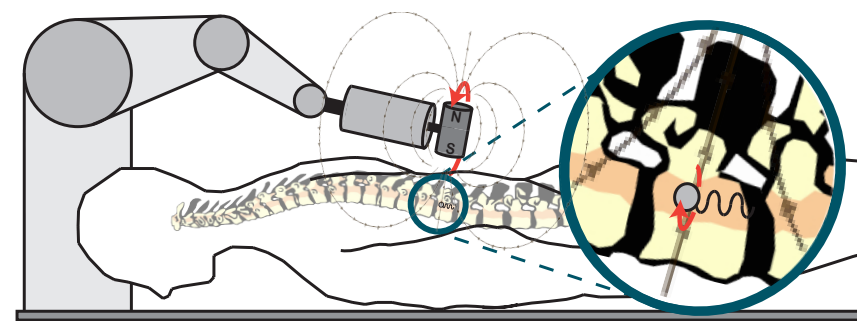

Fig. 1. Conceptual image of a microrobot (inset) propelled through the spinal canal using a rotating-permanent-magnet manipulator.

as using the microrobots to tow guidewires [7]. They have also explored crawling through lumens (e.g. blood vessels) by pressing the helical structure against the lumen's walls [8]. The devices developed by the Tohoku group are a few millimeters in size. A group at ETH Zurich developed the first microscopic magnetic helical swimmers, with a length of approximately $30 \mu \mathrm{m}$ [9], [10]. The "Artificial Bacterial Flagella" consist of a self-scrolling nanocoil propeller attached to a thin-square-plate "head" made of soft-magnetic material. Recently, a group at Harvard University demonstrated the batch fabrication and control of microswimmers that are even smaller, with a length of approximately $1 \mu \mathrm{m}$ [11].

For medical applications, helical microrobots are promising for use in open fluid, lumens (i.e. tubes), and softtissue environments (Fig.1). Helical microrobots also have the potential to smoothly transition between different environments. It has been proposed that a strip of drug or some other payload can be twisted dynamically into a helical structure under applied torsion, such that the payload of the microrobot is itself the propeller [12]. For movement through soft tissues, helical propulsion may be the only viable option for an untethered microrobot. In addition, Abbott et al. recently concluded that helical propulsion becomes desirable compared to other competing magnetic-propulsion methods as the device is scaled down, if one considers the geometry of the human body and the practical limitations in generating strong controlled magnetic fields [13].

In all prior work in magnetic helical microrobots, the magnetic-field-generation system is constructed as an orthogonal arrangement of electromagnetic coil pairs, which are used to generate rotating magnetic fields at the center of the system's workspace. This type of system results in very simple control because each coil pair is responsible for the magnetic field in only one direction, such that three coil pairs can be used to create a 3-D magnetic field vector in any direction, and the uniformity of the field at the center of the workspace creates a pure magnetic torque, which is used to 
rotate the microrobot without any undesirable forces due to field gradients. Although uniform-field systems have proven to be effective under a microscope and in other laboratory settings, they are difficult to scale up to the size required for in vivo medical devices. A reliance on uniform fields requires that the microrobot be located in the small central region of the system's workspace. However, there are no locations in the human body that can be described as being "at the center" in any reasonable sense; the human body is geometrically too complex. Consider the microrobot swimming up the spinal canal in Fig. 1: the spine is located much closer to the back of the patient than to the front, and it is very difficult to truly surround the microrobot in the direction along the length of the spine (the torso is a significant obstacle). For many other systems in the body (e.g. the urinary system, the brain, an eye), it is also possible to get very close if one approaches from certain, but not all, directions.

We propose the use of nonuniform magnetic fields emanating from a single rotating permanent magnet for control of helical microrobots. Allowing nonuniform magnetic fields makes it possible to place the magnet closer to the patient, which permits the use of a smaller and less-expensive systems. The use of nonuniform fields results in undesirable field-gradient forces, making control more challenging, but may ultimately result in superior systems-in terms of size and cost-compared to using uniform fields. What is not yet clear is how the performance of the proposed helical-propulsion method will compare to proven gradientpulling methods [14] at the relatively large scales of interest $(\sim 1 \mathrm{~mm})$ for many medical application [1]. In this paper, we present the proposed concept, and we show preliminary experimental and theoretical results that indicate that this is a wireless control method warranting further investigation.

\section{Magnetic Helical Microrobots}

Propulsion of magnetic helical microrobots swimming along their axes is well understood [13]. The sum of applied nonfluidic forces $f$ and torques $\tau$ that act on a helical microrobot are linearly related to its linear-axial velocity $v$ and rotational velocity $\omega$ by a propulsion matrix:

$$
\left[\begin{array}{l}
v \\
\tau
\end{array}\right]=\left[\begin{array}{cc}
a & b \\
-b & c
\end{array}\right]\left[\begin{array}{l}
f \\
\omega
\end{array}\right]
$$

The parameters $a, b$, and $c$ encapsulate the geometric and environmental properties of the microrobot.

With magnetic helical microrobots, an applied magnetic field $\mathbf{B}$ at the location of the microrobot is transduced into torque $\mathbf{T}$ as

$$
\mathbf{T}=\mathbf{m} \times \mathbf{B}
$$

where $\mathbf{m}$ is the dipole strength of the magnet rigidly attached to the microrobot perpendicularly to the axis of the helix [15]. The component of $\mathbf{T}$ on the helix axis is $\tau$ in (1). Any spatial gradient in the applied field at the location of the microrobot is transduced into applied forces as

$$
\mathbf{F}=(\mathbf{m} \cdot \nabla) \mathbf{B}
$$

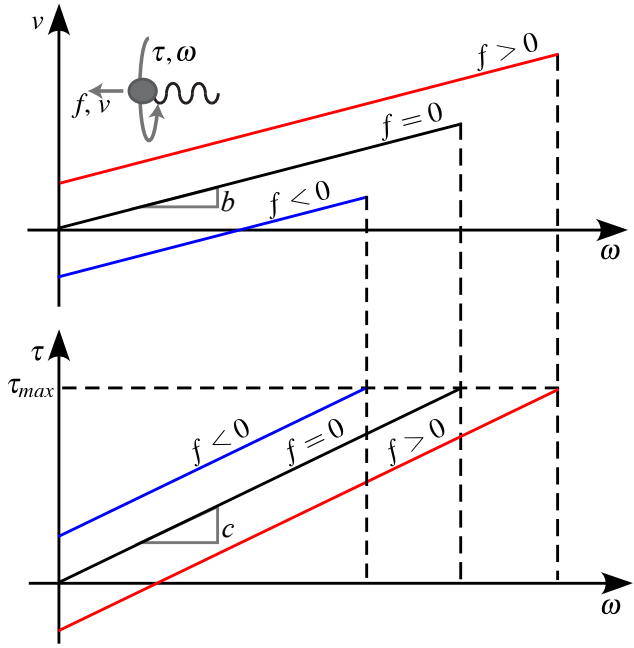

Fig. 2. Qualitative behavior of magnetic-helical-microrobot propulsion, based on [13]. Parameters are defined in (1). Sign convention is defined in the inset.

The component of $\mathbf{F}$ on the helix axis is $f$ in (1).

The rotation frequency of the magnetic field is the fundamental control input. The microrobot rotates in sync with the applied field, nearly instantaneously reaching an equilibrium phase shift such that the magnetic torque (2) counterbalances the rotational fluidic drag torque. The second input to the system is the sum of applied nonfluidic forces, $f$, which includes the microrobot's weight, forces due to magnetic field gradients (3), and any other loads.

Fig. 2 shows the behavior observed with this type of propulsion. The forward velocity grows linearly with frequency until a "step-out" frequency is reached. Beyond this step-out frequency, the available magnetic torque is no longer sufficient to keep the microrobot rotating in sync with the applied field, resulting in a drastic decrease in velocity. This step-out behavior has been experimentally observed at a wide range of scales [4], [10].

As stated earlier, all prior demonstrations of magnetic helical microrobots have utilized orthogonal arrangements of electromagnetic coil pairs, which are used to generate rotating uniform magnetic fields at the center of the system's workspace (i.e. $\mathbf{F}=\mathbf{0}$ in (3)). This method is difficult to scale up to the size required for in vivo medical devices [13], which is best explained by considering the magnitude of the field at the center of a given coil pair:

$$
|\mathbf{B}|=\frac{\mu_{0} R^{2} i}{\left(R^{2}+L^{2}\right)^{3 / 2}}
$$

where $\mu_{0}=4 \pi \times 10^{-7} \mathrm{~T} \cdot \mathrm{m} \cdot \mathrm{A}^{-1}$ is the permeability of free space, $i$ is the current flowing through the coils in $\{\mathrm{A}\}, R$ is the radius of the coils in $\{\mathrm{m}\}$, and the coils are separated by a distance $2 L\{\mathrm{~m}\}$. The field strength changes as $\sim L^{-3}$ and $\sim R^{-1}$, which means that if we make our coils big enough to fit around a human, it will be difficult to generate a strong magnetic field at the center. This challenge in scaling up for clinical use is fundamentally due to the difficulty in building orthogonal coil pairs that are large enough such that 
the region of interest inside the human body is located at the center of the orthogonal coil pairs, where the field is uniform. Removing the need for the microrobot to be at the center of the magnetic system's workspace will ultimately result in systems that better scale for in vivo devices.

\section{Control Using A \\ Rotating-Permanent-Magnet (RPM) MANipulator}

The nonuniform magnetic field emanating from a single rotating permanent magnet can be utilized to provide wireless propulsion to a helical microrobot. Using this technique, there are two fundamental control strategies: axial control, depicted in Fig. 3a,b, is control with the microrobot located along the rotation axis of the magnet; radial control, depicted in Fig. 3c,d, is control with the microrobot located perpendicular to the the magnet.

Reversing the microrobot's direction is accomplished simply by changing the rotation direction of the RPM manipulator. Steering is accomplished via remote-center-of-motion movements of the RPM manipulator about the microrobot, as depicted in Fig. 3a,c. During axial control steering, the RPM manipulator moves on a sphere enclosing the microrobot, such that the RPM manipulator's axis always points at the microrobot. During radial control steering, the RPM manipulator moves on a circle in the plane defined by the microrobot and the RPM manipulator's axis for one degree of freedom (DOF), and it rotates about the radial line (see Fig. 3c) for the other DOF. In both control strategies, the RPM manipulator's axis and the microrobot's axis will be parallel in steady state. Small steering inputs will result in the microrobot continually servoing to the desired steadystate orientation. Moving the RPM manipulator closer/farther from the microrobot results in a change in the magnitude of the applied field and field gradient at the microrobot, but does not result in any steering.

In the envisioned use, the RPM manipulator will be mounted on and position controlled with a multi-DOF manipulator (Fig. 1). It may also be possible for the RPM manipulator to be used as a hand-held tool by a clinician.

The field generated by the RPM manipulator can be modeled by a point-dipole model:

$$
\mathbf{B}(\mathbf{p})=\frac{\mu_{0}}{4 \pi|\mathbf{p}|^{3}}\left(\frac{3(\boldsymbol{\Gamma} \cdot \mathbf{p}) \mathbf{p}}{|\mathbf{p}|^{2}}-\mathbf{\Gamma}\right)
$$

where $\boldsymbol{\Gamma}$ is the RPM manipulator's magnetic dipole in $\left\{A \cdot m^{2}\right\}$, and $\mathbf{p}$ is the location of the microrobot with respect to the magnetic dipole in $\{\mathrm{m}\}$. This model is exact for the field of a spherical magnet, and it will approximate the far field of magnets of nonspherical geometry. The strength of the field decays as $\sim|\mathbf{p}|^{3}$, similar to electromagnetic coils. However, compared to electromagnetic coils, the RPM manipulator can be placed closer to the microrobot.

A detailed analysis of (2), (3), and (5) reveal that, in both the axial- and radial-control regions, the magnetic force on the microrobot vanishes when the magnetic torque is maximized (i.e. when $\mathbf{M} \cdot \mathbf{B}=0$ ). This is a very promising result, indicating that parasitic forces due to field gradients
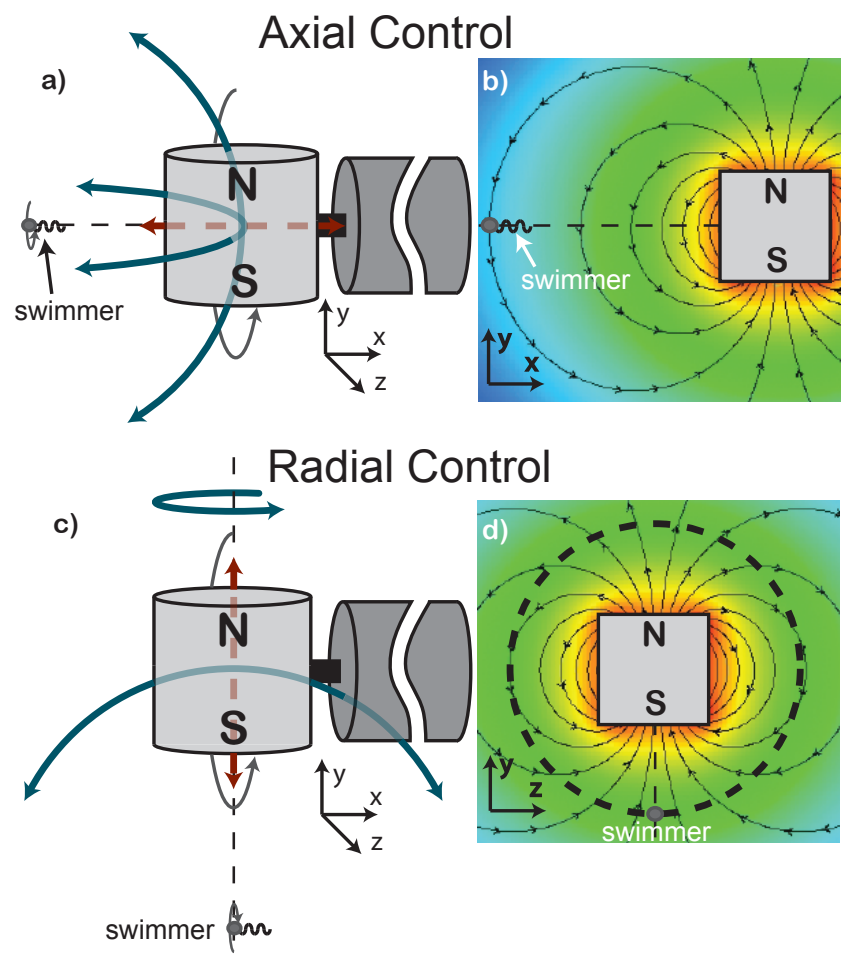

Fig. 3. Axial control and radial control of a magnetic helical microrobot with an RPM manipulator. (a) and (c) depict how steering is accomplished. Blue solid arrows indicate remote-center-of-motion movements that result in microrobot steering, and red dashed arrows indicate translations of the manipulator that result in no steering, but that change the field and fieldgradient values at the microrobot. (b) and (d) show magnetic field plots. The dashed circle in (d) is the region of the magnetic field that will affect the microrobot throughout the rotation cycle during radial control.

are minimized in the very condition where we achieve peak performance of the magnetic helical microrobot.

\section{Prototype Devices}

\section{A. Rotating-Permanent-Magnet (RPM) Manipulator}

The RPM manipulator, shown in Fig. 4, consists of a permanent magnet housed in Delrin, mounted on a Maxon DC Motor. A manipulator was designed to hold two different magnets: an axially magnetized cylindrical magnet $25.4 \mathrm{~mm}$ in length and $25.4 \mathrm{~mm}$ in diameter, and a diametrically magnetized cylindrical $\mathrm{NdFeB}$ magnet of the same dimensions. Both magnets have a $6 \mathrm{~mm}$ hole along the cylinder's axis. Both magnets are Grade- $42 \mathrm{NdFeB}$. Because the magnets have the same magnetized volume, we can perform a direct comparison of the different magnet geometries.

Magnetic field magnitudes were measured in both the axial- and radial-control regions, for both magnet types. Measurements were made using a Hirst GM08 gaussmeter, mounted on a 4-DOF (3-DOF translation, 1-DOF rotation) micromanipulation system constructed of modular Thorlabs MTS50 and PRM1 stages.

For the axial-control region, the magnitude of the field can be described by a simplification of (5), and the field gradient can be computed by differentiation:

$$
|\mathbf{B}|=\frac{\mu_{0}|\boldsymbol{\Gamma}|}{4 \pi|\mathbf{p}|^{3}} \quad, \quad \frac{d|\mathbf{B}|}{d|\mathbf{p}|}=-\frac{3 \mu_{0}|\boldsymbol{\Gamma}|}{4 \pi|\mathbf{p}|^{4}}
$$



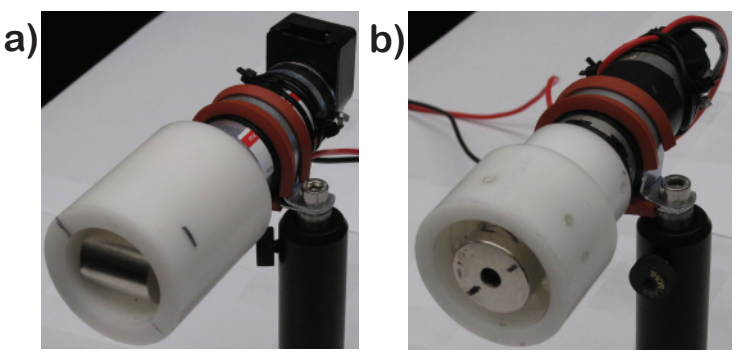

Fig. 4. The rotating-permanent-magnet (RPM) manipulator, consisting of (a) an axially magnetized magnet, and (b) a diametrically magnetized magnet, mounted on a DC motor. In both cases, the magnetization is perpendicular to the motor axis.

Fig. 5 shows the measured field data, along with the resulting point-dipole fit of (6), for each of the magnet types in the axial-control region. The dipole strengths, $|\boldsymbol{\Gamma}|$, from the fit were found to be $10.2 \mathrm{~A} \cdot \mathrm{m}^{2}$ and $12.6 \mathrm{~A} \cdot \mathrm{m}^{2}$ for the axial and diametric magnets, respectively. Clearly, the point-dipole model is a good approximation of the measured data in the region shown. The data indicate that the field of the diametrically magnetized magnet is stronger than that of the axially magnetized magnet at a given distance.

Fig. 6 shows measured data for the radial-control region at two different radial distances (each corresponding to a circle of data similar to the dashed line shown in Fig. 3d). Unlike axial control, during radial control the magnitude of the applied field varies by a factor of 2 throughout the cycle. Although it is less pronounced than with the axial-control data, the field of the diametrically magnetized magnet is again stronger than that of the axially magnetized magnet.

The choice of matching volumes in the two magnet types facilitated a direct comparison of their fields, but matching volumes is somewhat artificial. In the case of the diametrically magnetized magnet in Fig. 4b, the total volume inside the Delrin housing was not utilized. The magnet could be increase in size by $50 \%$ in the magnetization direction without changing the size of the Delrin housing. This would result in a factor of 2.25 increase in volume, which would lead to more than a factor of 2.25 increase in dipole strength (due to the increased aspect ratio of the magnet, which reduces internal demagnetizing fields). A diametrically magnetized magnet is the best choice for an RPM manipulator, since it better utilizes the volume of the rotating circular housing. However, the axially magnetized magnet is used in Section $\mathrm{V}$ because the diametrically magnetized prototype was only constructed and analyzed after the completion of these experiments.

\section{B. Helical Swimmers}

Several variants of up-scaled magnetic helical microrobots were constructed as shown in Fig. 7. The microrobot magnet used is a $1.625 \mathrm{~mm}$ diameter, $3.175 \mathrm{~mm}$ long, axially magnetized cylindrical $\mathrm{NdFeB}$ magnet. To measure the dipole strength $|\mathbf{m}|$ of this magnet, the maximum wirelessly applied torque was measured by mounting the magnet on an ATI Nano17 six-axis force/torque sensor, and placing the sensor at three locations in the applied field of the axially

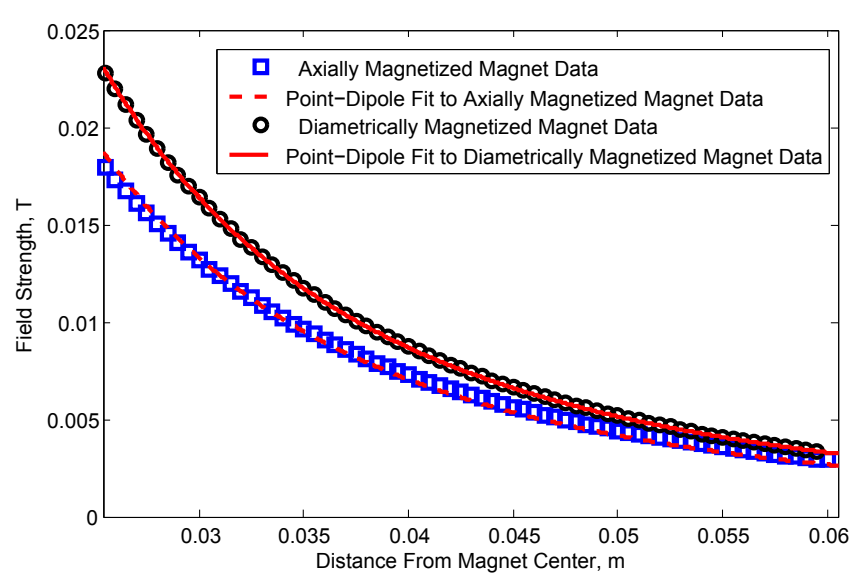

Fig. 5. Magnetic field measurements in the axial-control region, along with fitted point-dipole models, for the axially and diametrically magnetized magnets.

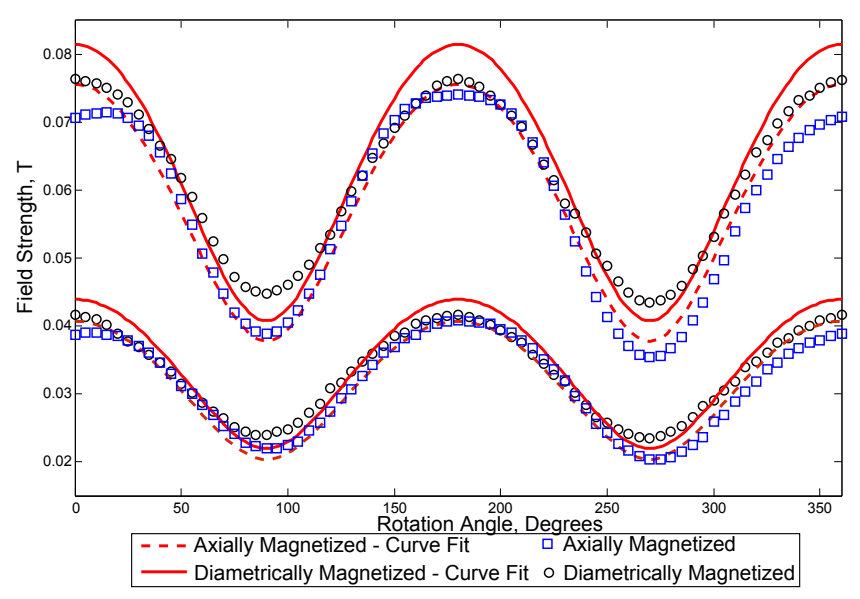

Fig. 6. Magnetic field measurements in the radial-control region for the axially and diametrically magnetized magnets. Data is shown for two different radial distances, similar to the dashed circle shown in Fig. 3d: the upper sets correspond to a distance from the magnet's rotation axis of $30.5 \mathrm{~mm}$, and the lower sets correspond to a distance of $38.1 \mathrm{~mm}$. The point-dipole models shown are those obtained from the data sets in Fig. 5.

magnetized RPM manipulator magnet. This maximum torque occurs at a lead angle of $90^{\circ}$, as described in (2), between the manipulator magnet and microrobot magnet. The dipole moment is calculated according to (2) using the values of $|\mathbf{B}|$ shown in Fig. 5. At distances of $25 \mathrm{~mm}, 35 \mathrm{~mm}$, and $45 \mathrm{~mm}$, the maximum torque was measured as $0.41 \mathrm{mN}$ $\mathrm{mm}, 0.13 \mathrm{mN}-\mathrm{mm}$, and $0.069 \mathrm{mN}-\mathrm{mm}$, respectively. With applied field magnitudes of $54 \mathrm{mT}, 20 \mathrm{mT}$, and $9.2 \mathrm{mT}$, we calculated the $|\mathbf{m}|$ in the range $6.9-7.5 \mathrm{~mA} \cdot \mathrm{m}^{2}$, with an average of $7.2 \mathrm{~mA} \cdot \mathrm{m}^{2}$. Additionally, force measurements due to field gradients were taken at the same three distances for a $360^{\circ}$ rotation of the manipulator magnet with the microrobot magnet fixed. The gradient force goes to zero at the instant when torque is maximized: at a lead angle of $90^{\circ}$. From this result, which is supported by analysis, we can deduce that running near step-out will result in similar propulsion as that of a uniform magnetic field (i.e. $\mathbf{F}=\mathbf{0}$ in (3)). 


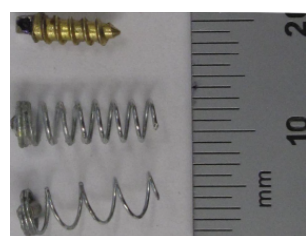

Fig. 7. Up-scaled microrobot prototypes manufactured from springs and cylindrical ( $3.175 \mathrm{~mm}$ length, $1.625 \mathrm{~mm}$ diameter) $\mathrm{NdFeB}$ magnets. The lowest "microrobot" is used in all experimentation.

\section{EXPERIMENTAL RESULTS AND DISCUSSION}

Experiments were conducted to verify the ability to propel the microrobot away from the manipulator magnet, as swimming toward the manipulator is always easier due to attractive field-gradient forces. In all experiments, the RPM manipulator with the axially magnetized magnet (Fig. 4a) is used to propel the lower microrobot of Fig. 7, which has a diameter of $4.1 \mathrm{~mm}$, through a $4.76 \mathrm{~mm}$-inner-diameter Tygon tube. The tubes are filled with water because the swimmer prototypes are not much bigger than the 1-mmdiameter devices envisioned for biomedical use. A more viscous fluid could be used to simulate the low-Reynoldsnumber environment of microswimmers, and performance would likely improve.

\section{A. Axial Control}

The experiments shown in Fig. 8 correspond to Fig. 3a,b. During axial control, we observe two phenomena of interest. One is step-out: the frequency above which the microrobot can no longer rotate continuously in sync with the RPM manipulator. Step-out is well understood for magnetic helical microrobots in uniform fields, as described in Section II. In the axial-control region, the step-out frequency is a function of the field magnitude, which is a function of distance from the manipulator magnet as in (6). The other observed phenomenon, which is unique to control with nonuniform magnetic fields, is what we call break-away: the frequency above which the microrobot produces enough propulsion to overcome the attractive gradient force and move away from the manipulator. During axial control, the microrobot should always run below the step-out frequency, and when moving away from the RPM manipulator, the microrobot must run above the break-away frequency. Experiments to determine the break-away and step-out distances for varying rotational frequencies of a microrobot being propelled away from the RPM manipulator are presented in Fig. 9.

At high rotation frequencies, break-away occurs close to the manipulator. However, the microrobot is not capable of making much progress before step-out. At low rotation frequencies there is less propulsion, so the distance from the manipulator to the microrobot must be farther to break away from field-gradient forces. However, this results in the microrobot being capable of swimming farther distances.

If we consider the field and field gradient of (6), and realize that the magnitude of the dipole strength $\Gamma$ is proportional to volume, we find that the field changes homothetically, meaning that it is scale-invariant, but that the gradient has the
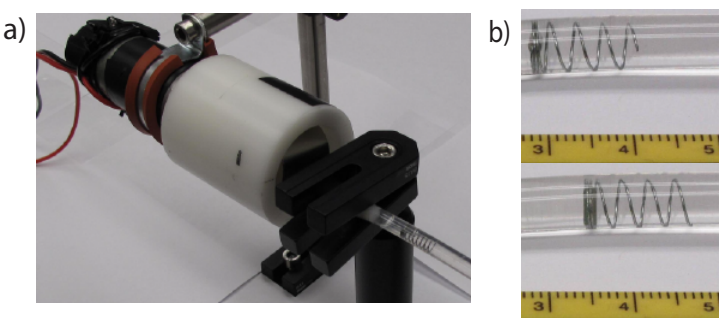

Fig. 8. a) Experimental setup for axial control. b) Microrobot swimming in a plastic lumen via propulsion from axial control.

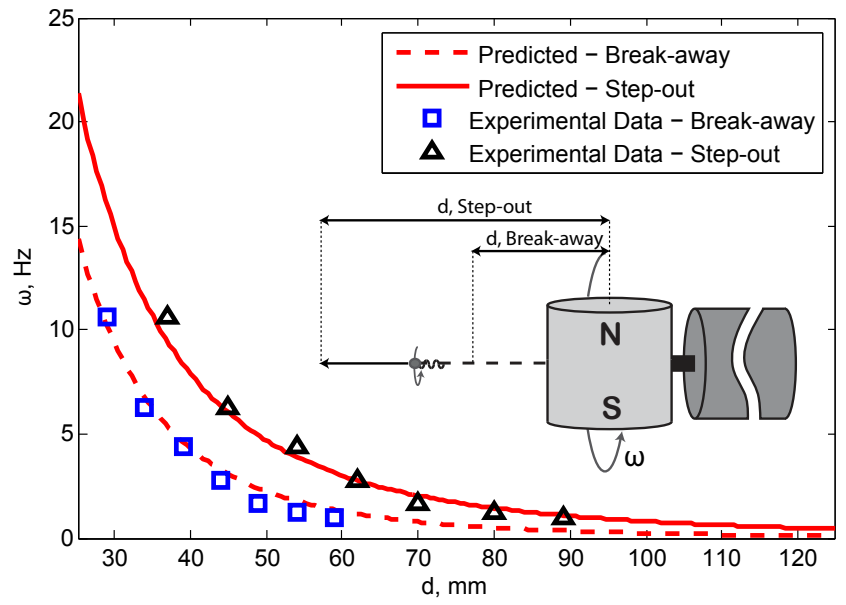

Fig. 9. Break-away and step-out of a microrobot under axial control. To be propelled away from the manipulator, the microrobot must operate between the two curves.

effect of decreasing as we increase the scale of the system. That is, bigger magnets can project a given field magnitude farther, with an accompanying reduction of field gradient at that given field magnitude.

In Fig. 10 we show a theoretical plot analogous to Fig. 9. We consider the step-out and break-away curves for the axially magnetized RPM manipulator magnet, for the same magnet if its length scale is enlarged by a factor of 2 , and for a microrobot whose length scale is shrunk by a factor of 2 . The distances are nondimensionalized with respect to the size of the RPM manipulator magnet. We find that the step-out curve is unchanged with scaling, indicating that increasing the length scale of the manipulator magnet by a factor $X$ will allow the step-out distance to be projected farther by a factor $X$ for the same frequency. Scaling of the microrobot has no effect on step-out. We find that the break-away curve does change: the effect of either increasing the length scale of the manipulator magnet by a factor $X$ or decreasing the length scale of the microrobot by the same factor $X$ is to broaden the working region equivalently, such that larger distances can be traveled by the microrobot at a given frequency, and a wider range of frequencies will work for a given distance from the manipulator.

\section{B. Radial Control}

The experiments shown in Fig. 11 correspond to Fig. 3c,d, as well as Fig. 1. The microrobot was placed directly beneath the RPM manipulator and allowed to swim in either 


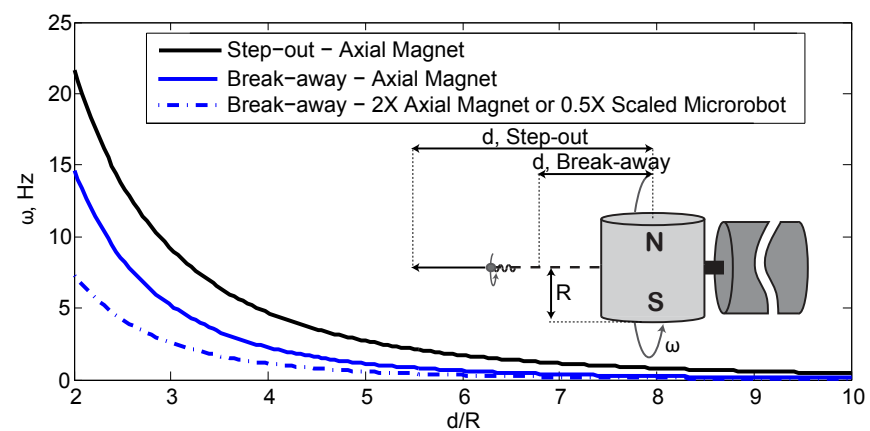

Fig. 10. Effect of RPM manipulator and microrobot scaling on step-out and break-away during axial control. The distance of the microrobot is normalized by the size of the RPM manipulator's magnet.

direction, forward or reverse. The natural tendency is for the microrobot's magnet to align itself below the manipulator's magnet, where there is a local minimum in the field-gradient forces along the lumen. There are also attractive forces that pull the microrobot laterally into the wall of the lumen. Here, we consider the ability to propel the microrobot away from the equilibrium position. Due to the local minimum in the attractive force, there is no break-away frequency; the microrobot can be propelled at any frequency. During the experiment, the microrobot is propelled at a constant frequency until step-out is observed, as shown in Fig. 12. We do not yet have a good analytical model for this behavior.

\section{CONClusion And Future Work}

We presented a rotating-permanent-magnet (RPM) manipulator as a viable option for wireless control of magnetic helical microrobots. For control in two distinct regions of the RPM manipulator, the microrobot can be propelled both toward and away from the manipulator, overcoming attractive forces due to gradients in the nonuniform magnetic field. Propulsion in the axial-control region is predictable, and has been experimentally verified. Radial control is more complicated due to variations in the magnetic field and field gradient that occur throughout the rotation cycle. A complete understanding of this control scenario requires additional modeling and experimentation. We concluded that the RPM manipulator should use a diametrically magnetized magnet.

This paper focused primarily on 1-DOF propulsion in lumens, which is representative of a number of potential medical procedures where a microrobot might be utilized. In the future we will consider open swimming, as well as propulsion through soft tissue. Preliminary investigation has shown that the proposed steering method works well for gradual (large radius) steering maneuvers, but that attempting to make tight turns results in unpredicted and nonintuitive motions of the microrobot. Swimming models for the complex scenario of swimming in 3-D using nonuniform magnetic fields must still be developed.

\section{REFERENCES}

[1] B. J. Nelson, I. K. Kaliakatsos, and J. J. Abbott. Wireless microrobots for minimally invasive medicine. Annual Review of Biomedical Engineering, To appear in 2010.
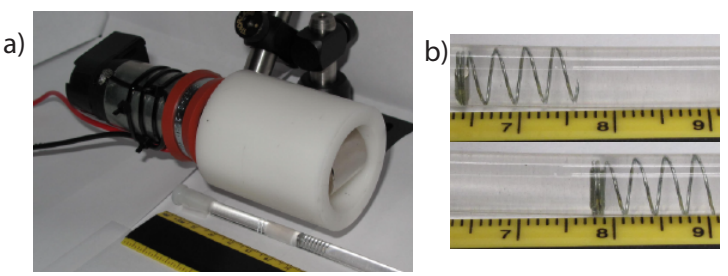

Fig. 11. a) Experimental setup for radial control region. b) Microrobot swimming via propulsion from radial control

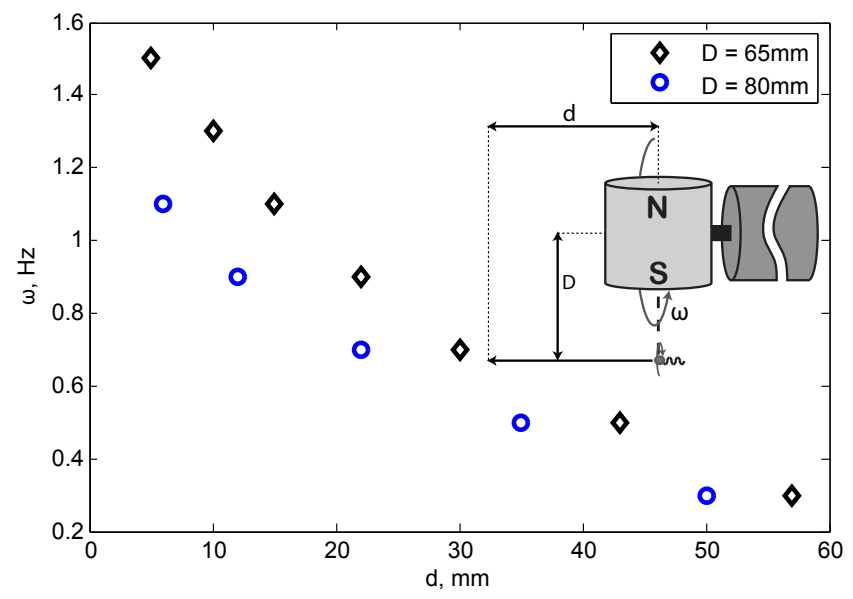

Fig. 12. Experimental data of step-out of a microrobot under radial control.

[2] E. M. Purcell. Life at low reynolds number. American J. Physics, 45(1):3-11, 1977.

[3] T. Honda, K. I. Arai, and K. Ishiyama. Micro swimming mechanisms propelled by external magnetic fields. IEEE Trans. Magnetics, 32(5):5085-5087, 1996.

[4] K. Ishiyama, K. I. Arai, M. Sendoh, and A. Yamazaki. Spiraltype micro-machine for medical applications. J. Micromechatronics, 2(1):77-86, 2003.

[5] M. Sendoh, K. Ishiyama, K. I. Arai, M. Jojo, F. Sato, and M. Matsuki. Fabrication of magnetic micromachine for local hyperthermia. IEEE Trans. Magnetics, 38(5):3359-3361, 2002.

[6] F. Sato, M. Jojo, H. Matsuki, T. Sato, M. Sendoh, K. Ishiyama, and K. I. Arai. The operation of a magnetic micromachine for hyperthermia and its exothermic characteristic. IEEE Trans. Magnetics, 38(5):33623364, 2002.

[7] K. Kikuchi, A. Yamazaki, M. Sendoh, K. Ishiyama, and K. I. Arai. Fabrication of a spiral type magnetic micromachine for trailing a wire. IEEE Trans. Magnetics, 41(10):4012-4014, 2005.

[8] M. Sendoh, K. Ishiyama, and K.-I. Arai. Fabrication of magnetic actuator for use in a capsule endoscope. IEEE Trans. Magnetics, 39(5):3232-3234, 2003.

[9] L. Zhang, J. J. Abbott, L. X. Dong, B. E. Kratochvil, D. Bell, and B. J. Nelson. Artificial bacterial flagella: Fabrication and magnetic control. Applied Physics Lett., 94(064107), 2009.

[10] L. Zhang, J. J. Abbott, L. X. Dong, K. E. Peyer, B. E. Kratochvil, H. Zhang, and B. J. Nelson. Characterizing the swimming properties of artificial bacterial flagella. Nano Lett., 9(10):3663-3667, 2009.

[11] A. Ghosh and P. Fischer. Controlled propulsion of artificial magnetic nanostructured propellers. Nano Lett., 9(6):2243-2245, 2009.

[12] H. Li, J. Tan, and M. Zhang. Dynamics modeling and analysis of a swimming microrobot for controlled drug delivery. In Proc. IEEE Int. Conf. Robotics and Automation, pages 1768-1773, 2006.

[13] J. J. Abbott, K. E. Peyer, M. Cosentino Lagomarsino, L. Zhang, L. X. Dong, I. K. Kaliakatsos, and B. J. Nelson. How should microrobots swim? Int. J. Robotics Research, 28(11-12):1434-1447, 2009.

[14] S. Martel, J.-B. Mathieu, O. Felfoul, A. Chanu, E. Aboussouan, S. Tamaz, and P. Pouponneau. Automatic navigation of an untethered device in the artery of a living animal using a conventional clinical magnetic resonance imaging system. Applied Physics Lett., 90(11):114105(1-3), 2007.

[15] E. P. Furlani. Permanent Magnet and Electromechanical Devices. Academic Press, San Diego, California, 2001. 